\title{
CORRIGENDUM
}

\section{Within Group and Between Group Variation Expected in Human Behavioral Characters ${ }^{1}$}

\section{Gordon Allen ${ }^{2}$}

On page 191 appeared the statement, "Preference for, or avoidance of consanguineous matings within an isolate does not, like random inbreeding, have a permanent effect on the store of genetic variability." This is only approximately correct, and so a footnote was appended. The footnote was, however, even less accurate. The present note, again avoiding mathematical expressions, hopefully provides a more accurate explanation.

Genetic drift ("random inbreeding") can theoretically be measured within a defined population and time interval by the increase in the average inbreeding coefficient $(F)$ between the initial and final generations, provided that both of these generations are produced by random mating. The decrease in heterozygosity implied by the increasing values of $F$ constitutes a loss of genetic diversity through reduction in frequency of alleles below the value of 0.5 .

In contrast, deviation from random mating within a population ("systematic inbreeding") alters the average inbreeding coefficient without directly altering either gene frequencies or the diversity of alleles. If, however, the isolate is small enough to manifest genetic drift at the same time, then the mating system will affect the rate of drift, thus having a delayed and indirect effect on the diversity of alleles.

In more detail, systematic inbreeding has two opposite effects on the rate of drift, which may sometimes exactly offset each other. Preference for consanguineous matings (positive systematic inbreeding) accentuates errors of sampling from the gene pool that occur in survival and reproduction of zygotes and individuals. On the other hand, by minimizing the number of

\footnotetext{
${ }_{1}^{1}$ This paper appeared in Behavior Genetics 1 (3/4): 175-194 (1970).

2 National Institute of Mental Health, Public Health Service, Bethesda, Maryland.
} 
heterozygous loci, it reduces the sampling errors in chance survival and reproduction of gametes from each reproducing individual. Kimura and Crow [Genet. Res. 4: 399 (1963)] showed that, if the first type of sampling error (in individual reproduction) can be avoided, reserve variability is best protected by creating multiple inbred lines in which there is $n o$ heterozygosity. But Macluer and Schull [Am. J. Human Genet. 22: 176 (1970)] showed that, in a human population that does not become subdivided and in which both zygotes and gametes are sampled, preferred consanguineous mating (positive inbreeding) has the net effect of increasing the rate of genetic drift (gene loss). 